\title{
FREELY-MIGRATING DEFECTS: THEIR PRODUCTION AND INTERACTION WITH CASCADE REMNANTS*
}

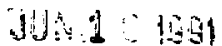

\author{
L. E. Rehn and H. Wiedersich \\ Materials Science Division \\ Argonne National Laboratory \\ Argonne 1160439 USA \\ ANL $/ \mathrm{CP}--72201$ \\ DE91 013618
}

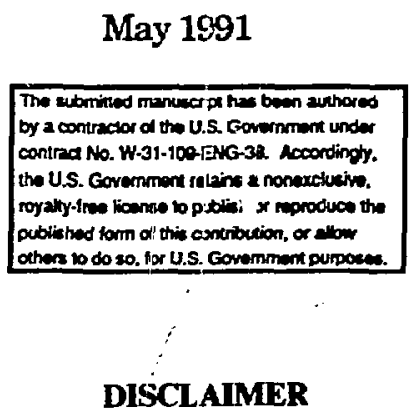

This report was prepared as an account of work sponsored by an agency of the United States Government. Neither the United States Government nor any agency thereof, nor any of their employees, makes any warranty, express or inplied, or assumes any legal liability or responsibility' for the accuracy, completeness, or usefulness of any information, apparal us, product, or process disclosed, or represents that its use would not infriage privately owned rights. Reference herein to any specific commercial product, process, or service by trade aame, trademark, manufacturer, or otherwise does not necessarily constitute or imply its endorsement, recommendation, or favoring by the United States Government or any agency thereof. The views and opinious of authors expressed berein do not necessarily state of reflect those of the United States Government or any agency the:reof.

Invited paper submitted to the "International Conference on Physics of Irradiation Effects in Metals", to be held in Siofok, Hungary, May 20-24, 1991.

\section{*Work supported by the U. S. Departraent of Energy, BES-Materials Sciences, under Contract W-31-109-Eng-38.}

\section{DISCLAIMER}

\begin{abstract}
This report was prepared as an account of work sponsored by an agency of the United States Government. Neither the United States Government nor any agency thereof, nor any of their employes, makes any warranty, express or inplied, or assumes any legal liability or responsibility for the accuracy, completeness, or usefulness of any information, apparatus, product, or process disclosed, or represents that its use would not inftinge privately owned rights. Reference herein to any specific commercial product, process, or service by trade name, trademark, manufacturer, or otherwise does not necessarily constitute or imply its endorsement, recommendation, or favoring by the United States Government or any agency thereof. The views and opinions of authors expressed herein clo not necessarily state or reflect those of the United States Government or any agency thereol:
\end{abstract}


FREELY-MIGRATING DEFECTS: THEIR PRODUCTION AND INTERACTION WITH CASCADE REMNANTS.* I. E. REHN and H. WIEDERSICH, Materials Science Division, Argonne National Laboratory, Argonne IL 60439 USA

\begin{abstract}
Many microstructural changes that occur during irradiation are driven primarily by freely-migrating defects, $i$. e. those defects which escape from nascent cascades to migrate over distances that are large relative to typical cascade dimensions. Several measurements during irradiation at elevated temperatures have shown that the survival rate of freely-migrating defects decreases much more strongly with increasing primary recoil energy than does the survival rate for defects generated at liquid helium temperatures. For typical fission or fusion recoil spectra, and for heavy-ion bombardment, the fraction of defects that migrate long-distances is apparently only $\sim 1 \%$ of the calculated dpa. This small surviving fraction of freely-migrating defects results at least partially from additional intracascade recombination at elevated temperatures. However, cascade remnants, e.g., vacancy and interstitial clusters, also contribute by enhancing intercascade defect annihilation. A recently developed rate-theory approach is used to discuss the relative importance of intra- and intercascade recombination to the survival rate of freely-migrating defects. Within the validity of certain simplifying assumptions, the additional sink density provided by defect clusters produced directly within individual cascades can explain the difference between a defect survival rate of about $30 \%$ for low dose, low temperature irradiations with heavy jons, and a survival rate of only $-1 \%$ for freely-migrating defects at elevated temperatures. The status of our current understanding of freely. migrating defects, including remaining unanswered questions, is also discussed.
\end{abstract}

*Work supported by the U.S. DOE BES-Materials Sciences under Contract \#W-31-109ENG-8 


\section{INTRODUCTION}

For several decades, attempts to correlate property changes in irradiation environments with different characteristic recoil spectra, e.g., fission, fusion, and thermal neutrons, as well as high-energy ion and electron sources, have focussed on calculations of the fraction of displaced atoms, or dpa, produced by the different sources. Although normalizing property changes on the basis of dpa generally improves any previously recognized trend, this approach fails far short of obtaining a quantitative correlation [1-3]. The reason for this failure is widely recognized. Whereas dpa calculations can be used to obtain the "total" number of defects generated by different sources, many microstructural changes that occur during irradiation are driven primarily by only the freely-migrating defects, i.e., those defects which escape from displacement cascades to migrate over distances that are large relative to nascent cascade dimensions [4]. Such microstructural changes include void swelling, radiation-induced segregation, radiation-enhanced diffusion, and as has been recognized more recently, at least some degree of radiation embrittlement [5]. Since the fraction of freely-migrating defects depends strongly on the spatial distribution of the generated defects, and since dpa does not take this spatial distribution into account, normalizing on the basis of dpa cannot be expected to produce quantitative property correlations between irradiation environments with substantially different primary recoil spectra.

About fifteen years ago, low-temperature measurements of defect preduction demonstrated that the fraction of surviving defects was a strong function of primary recoil energy [6]. For recoil energies $>20 \mathrm{keV}$, only $\leq 30 \%$ of the calculated number of defects appeared to survive the residual agitation occurring during the cascade cooling phase [7]. These measurements thus provided a first-order correction to the dpa normalization. However, the $30 \%$ correction produced only a slight improvement in correlating the types of microstructural changes listed above.

About ten years later evidence began to accumulate, primarily from experiments at elevated temperatures, that irradiations which generate energetically dense cascades are in fact much less than $30 \%$ as effective as light-ion, $\mathrm{MeV}$ electron, or thermal neutron irradiations at producing freely-migrating defects [4]. A subsequent systematic study of radiation-induced segregation rates in three different binary alloys yielded a dependence of freely-migrating defect production on primary recoil energy that is qualitatively similar to the measurements of defect survival at cryogenic temperatures [8-10]. However, the total fraction of defects found to undergo free migration at elevated temperatures for higher recoil energies is much smaller than the fraction that survives at low temperatures. For fission or fusion neutron irradiations, with typical recoil energies $>20 \mathrm{keV}$, the fraction of freely-migrating defects becomes $\leqslant 1 \%$ of the calculated dpa.

The importance of understanding the role of freely-migrating defects in irradiationinduced microstructural development has been recognized, and in fact stressed, in the summary reports of three recent workshops dealing with current areas of irradiation effects research $[2,3,11]$. Renewed interest in freely-migrating defects also occurred when surveillance measurements at the Oak Ridge High Flux Isotope Reactor (HFIR) demonstrated an unanticipated, order of magnitude enhancement in the embrittlement rate at the pressure vessel wall [12]. This enhancement was attributed to a strong 
neutron spectrum effect, i.e., a high thermal-to-fast neutron flux ratio, which created a larger than anticipated fraction of freely-migrating defects [5]. This discovery led to a lengthy shut down of HFIR, and the engineering implications of enhanced embrittlement for other highly thermalized neutron environments, e.g. components remote from the core, remains a topic of considerable current interest.

The production of freely-migrating defects has been reviewed in detail previously [4], and an update and further relevant information can be found in the recently published proceedings of two international workshops $[2,3]$. For these reasons, the following approach is taken in the present manuscript. Information obtained from lowtemperature studies on surviving defect production, and from primarily elevatedtemperature studies on freely-migrating defect production, is briefly summarized in Section II. In Section III, a recent proposal [13] that freely-migrating defect production correlates more strongly with nuclear stopping power than with primary recoil energy is discussed, and the advantages of using the latter quantity are elucidated. After this brief digression, we return to the dramatic decrease in freely-migrating defect production with increasing recoil energy in Section IV, discusing the potential impact of cascade remnants, i.e. vacancy and interstitial cluster formation, on the freelymigrating fraction [14]. Section V includes a summary of cur current understanding of freely-migrating defects, and a discussion of several rernaining unanswered questions.

\section{DEFECT SURVIVAL VS. FREELY-MIGRATING DEFECT PRODUCTION}

An overview [7] of damage efficiency measurements performed at cryogenic temperatures during electron, ion and neutron irradiation is given in Fig. 1. These lowtemperature studies provide information on the primary damage state $[15,16]$, i.e. the defect configurations that survive the cascade cooling phase. Low temperatures are employed in order to minimize the migration of defects subsequent to production, thus maximizing the fraction that survives.

The "defect-weighted" average recoil energy (that energy above and below which half the defects are produced) [6] is plotted as the abscissa for Fig. 1; this quantity is sometimes referred to as the "median recoil energy" [7]. The experimental data reveal a strong decrease in defect survival with increasing recoil energy from just above threshold to recoil energies of a few $\mathrm{keV}$. Since individual recoils of $1 \mathrm{keV}$ produce on average only about 10-20 Frenkel pairs, it can perhaps be considered surprising that this rapid drop occurs so early. At high recoil energies, greater than about 100 times threshold, the efficiency appears to saturate at about one-fourth the value found just above threshold. The observed tendency toward saturation demonstrates that the energy density within cascades does not increase indefinitely with increasing recoil energy, which is expected because of the increasing probability for subcascade formation [17]. The efficiency for neutrons (Fig. 1; squares) lies significantly below that for ions (triangles) with similar median recoil energies. As pointed out elsewhere [6,7], this occurs because the longrange Coulomb interaction with ions produces a low-energy tail in the recoil spectrum, where defect survival is enhanced.

The results of an extensive international effort to determine low-temperature defect production efficiencies in dilute alloys as well as pure metals have been tabulated by Jung et al. [18]. Dilute alloying additions ( $\leq 10 \%)$ produce no substantial effects during 
irradiation at low temperatures, where thermally activated defect migration is suppressed.

During irradiation, defect migration over distances large relative to cascade dimensions (typically 5-15 nm) occurs only at temperatures (typically $>0.4 \mathrm{~T}_{\mathrm{m}}$ ) where both vacancies and interstitials, and their small clusters, are mobile [19]. Experimental techniques with sufficient temporal $\left(\sim 10^{-11} \mathrm{~s}\right)$ and spatial resolution are not currently available for investigating the primary damage state at temperatures $(>50 \mathrm{~K})$ where one or more defect types are mobile. In fact, while a primary damage state for a cascade event can be clearly defined after a low-dose low-temperature irradiation, it is not clear that an analogous state even exists at temperatures where primary defects are mobile. Little information is available for extrapolating the low-temperature results to elevated temperatures, where some of the defects that were counted in the low-temperature studies (Fig. 1) will certainly be lost as a result of additional defect motion. Because local defect densities increase, the additional loss within cascades will also certainly increase with increasing primary recoil energy.

The fundamental quantity of interest at elevated temperatures is the number of freelymigrating defects. Rather than attempt any extrapolation of the low temperature results, many authors have taken a more direct approach to measuring freelymigrating defect concentrations. They select one of several property changes that result from freely-migrating defects and measure it for various steady-state or quasi steadystate irradiation conditions. Theoretical models are then used to extract the freelymigrating defect concentration. As has been discussed elsewhere [4], the qualitative agreement concerning freely-migrating defect concentrations between the different property measurements is generally quite good, but systematic quantitative information is rare.

Following this same strategy, we have used in situ measurements [8-10] of radiationinduced segregation (RIS) to determine the relative efficiencies of many different irradiation particles for producing freely-migrating defects. In agreement with simple theoretical arguments, the thickness of metastable $\mathrm{Ni}_{3} \mathrm{Si}$ surface coatings generated during irradiation was found to increase linearly with the square root of the irradiation dose. The RIS data also followed the predicted inverse-fourth-root dependence in the recombination-limited temperature regime, as well as the expected absence of a dose-rate dependence at higher temperatures, where the equilibrium vacancy concentration becomes rate controlling. Based upon the empirically determined and theoretically understood kinetic behavior, the efficiencies of the different ions for producing freelymigrating defects were extracted. These results, for three different alloy systems (Ni-Si, $\mathrm{Cu}-\mathrm{Au}$ and $\mathrm{Mo}-\mathrm{Re}$ ), are plotted in Fig. 2 as a function of the calculated average recoil energy.

The general shape of the freely-migrating defect efficiency curve in Fig. 2 is very similar to the low-temperature defect-survival results seen in Fig. 1. A strong decrease in tive efficiencies of both processes with increasing recoil energy occurs up to a few keV, and they both appear to saturate above $\sim 20 \mathrm{keV}$. The one major difference is that while the low-temperature defect production results saturate at about one-fourth the value found near threshold, the apparent saturation efficiency for freely migrating defects is considerably less, only a few to perhaps less than one per cent. The strong similarity in 
the functional dependence found for the three different alloy systems, and the general agreement found with the results from other authors [4] in a variety of pure materials, implies that this same functional dependence is a general characteristic of freelymigrating defect production in metals.

Wiedersich [20] has suggested the following simple empirical expression to describe the fraction of freely-migrating defects, $p(N)$, produced in cascades of various sizes:

$$
p(N)=\left(1-p_{\infty}\right) \exp \left[(1-N) / N_{d}\right]+p_{\infty} .
$$

Here, $\mathrm{N}$ is the number of defects produced in the cascade, $\mathrm{p}_{\infty}$ is the saturation fraction for high recoil energies, and $N_{d}$ is the characteristic decay length. Wiedersich has shown that the data in Fig. 2 can be fit quite well with values of $0.5 \%$ and 5 for $p_{\infty}$ and $\mathrm{N}_{\mathrm{d}}$, respectively.

There are several additional, although less systematic studies which support the low saturation value $(\sim 1 \%)$ for freely-migrating defect production. Among the more recent investigations, the Berlin group [21-23] has used SIMS to monitor radiation-enhanced diffusion of $\mathrm{Ni}$ in $\mathrm{Ni}$, in $\mathrm{Cu}$, and in $\mathrm{Fe}-20 \mathrm{Cr}-60 \mathrm{Ni}$ and $\mathrm{Fe}-20 \mathrm{Cr}-20 \mathrm{Ni}$, and obtained a value of $\left(1.5_{-1.2}^{+4.5}\right) \%$. Murphy and Perks [24] measured P segregation in Ni during irradiation with heavy ions and obtained saturation values of (1-10)\%. Heinisch [25] has compared tensile data obtained in a variety of neutron environments on pressure vessel steels and shown that a reasonable correlation can be obtained using the functional relationship exhibited by the data in Fig. 2. A listing and discussion of earlier results on freelymigrating defect production efficiencies can be found in Ref. 4.

\section{RECOIL ENERGY OR ENERGY LOSS}

The data in Figs. 1 and 2 have been plotted, as was done by the original authors, using calculations of the weighted-average recoil energy for the abscissa. Since the number of jumps a defect makes before annihilation depends strongly on whether the defects are produced in relatively isolated events or in cascade regions of locally high defect density, and since a partial description of this spatial arrangement can be inferred from the calculated distribution of energies with which the primary knock-on atoms recoil, a correlation of the measured efficiencies with median recoil energy is expected (and seen) for the data in Figs. 1 and 2. However, other parameters which reflect the degree of spatial correlation in defect production, e.g., maximum recoil energy or cascade energy density, might be selected instead. Indeed, the original authors of both data sets [6-8] have discussed the fact that no ideal choice is evident, particularly when correlations are sought between irradiations with very different primary types of particle-target interactions, e.g., ions (Coulombic) and fast neutrons (nuclear).

It has recently been suggested [13] that the nuclear stopping power also provides a reasonable, and perhaps even an improved correlation relative to the median recoil energy. The basis for this suggestion is contained in Fig. 3, where measured efficiencies of freely-migrating defect production have been plotted as a function of the average recoil energy (top figure), and of the nuclear stopping power (bottom figure), on a log-log scale. The MeV-ion data points were taken from Fig. 2; the 2-keV O and 300$\mathrm{keV} \mathrm{Ni}$ data are measurements from Refs. 13 and 22 . Fig. 3 suggests that nuclear 
stopping power, particularly with regard to the low-energy $\mathrm{O}^{+}$data, provides a better correlation with freely-migrating defect efficiency than does median recoil energy.

Before addressing this conclusion directly, there are several facets of Fig. 3 that require clarification. First, the high-energy $(\mathrm{MeV}) \mathrm{H}, \mathrm{He}, \mathrm{Li}$ and $\mathrm{Ni}$ data are actually relative fractions, not absolute as implied by the ordinate. Conversion to absolute numbers requires a benchmark, and the assumption made in drawing Fig. 3 is that the absolute fraction of freely-migrating defects is the same for the two $(300-\mathrm{keV}$ and $3-\mathrm{MeV}) \mathrm{Ni}$ irradiations, which appears reasonable in light of the apparent tendency toward saturation.

It should also be noted that there is no a priori reason requiring that the data exhibit log$\log$ behavior. In fact, the concept of subcascade formation implies a true saturation at high recoil energies, which is inconsistent with this assumption. In evaluating the appropriateness of a log-log plot, it is also important to consider the large uncertainty in the existing data (This will be discussed further in Section V.). The quoted [13] uncertainty of the $300-\mathrm{keV} \mathrm{Ni}$ and $2-\mathrm{keV} O$ data $\left(0.015_{-0.012}^{+0.045}\right)$ places both results within one standard deviation of the 2-MeV Li datum. Hence, even though the straight line passes quite close to both the $300-\mathrm{keV} \mathrm{Ni}$ and the $2-\mathrm{keV} \mathrm{O}$ results, these measurements clearly do not provide a stringent test of the log-log behavior assumed by drawing a straight line through the data.

The major distinction (Fig. 3 ) between plotting the data as a function of median recoil energy (top) or nuclear energy loss (bottom) occurs for the $2-\mathrm{keV} O$ datum. In addition to the large uncertainty, there are further aspects of the 2-keV $\mathrm{O}$ datum to consider. Ref. 13 assigns a value of $155 \mathrm{eV}$ for its median recoil energy. Considering that the range of a $2-\mathrm{keV} O$ ion in $\mathrm{Ni}$ is $\leq 3 \mathrm{~nm}$, i.e. less than a typical cascade dimension, intuitively it would appear more suitable to assign the entire deposited damage energy $(\sim 1 \mathrm{keV})$ as the recoil energy. Furthermore, the deposition of all the particle energy so near to the specimen surface (in fact a large part of it within one spontaneous recombination distance), will certainly cause a substantial loss of freely-migrating defects to this very effective defect sink.

From the above discussion, it is clear that the $2-\mathrm{keV} O$ result provides little useful information as to whether the median recoil energy or the energy loss provides a better correlation for freely-migrating defect production. In making a selection between different candidate parameters, there is at least one additional important factor to consider. One of the fundamental objectives of identifying a correlation parameter is for modeling irradiation-induced property changes in neutron (reactor) environments. In this regard, the energy loss per unit path length of a neutron (less than $10^{-2} \mathrm{eV} / \AA$ and therefore far to the left on the scale shown in Fig. 3) is clearly inappropriate for correlating freely-migrating defect production in neutron environments. Hence, the median recoil energy appears superior to nuclear stopping power for correlating defectinduced property changes in a wide variety of irradiation environments. 


\section{INTRA VS. INTERCASCADE DEFECT ANNIHILATION: THE ROLE OF CASCADE REIMINANTS}

The strong decrease that occurs in defect survival with increasing median recoil energy (Fig. 1) is straightforward to understand. According to the modified Kinchin and Pease expression [26,27], the number of atoms displaced by a recoiling atom is approximately equal to the recoil energy divided by $50-100 \mathrm{eV}$. Defect production as a function of primary recoil energy therefore develops in the following manner. As recoils grow in energy from tens to hundreds of electron volts, the introduction of randomly spaced Frenkel pairs gradually changes over to the generation of several defects in close proximity. Defect cascades can be clearly distinguished in computer-simulation studies for primary recoil energies above about $1 \mathrm{keV}[28,29]$, after which the size of the cascade region continues to increase with increasing energy. For recoils greater than a few tens of kilo-electron-volts, the rate of increase in spatial correlation among the defects slows because of the increasing probability for higher energy recoils to split into subcascades. Computer calculations have revealed that the amount of induced defect motion increases with increasing cascade energy, even to the extent of generating "liquid-like" cascade cores at sufficiently high deposited energy densities [30,31]. This additional induced defect motion, which enhances the probability of both annihilation and clustering of defects, is the reason for the strong decrease in defect survival with increasing recoil energy seen in Fig. 1.

Previously [4], the even stronger decrease in the fraction of freely-migrating defects with increasing recoil energy (Fig. 2) has been attributed to the same fundamental mechanism, i.e. defect motion within energetic cascade regions, which causes additional intracascade annihilation and clustering at elevated temperature. The two primary reasons for the intracascade interpretation were (1) the relatively wide temperature range over which the freely-migrating defect fraction was found to remain constant, and (2) the observation that the irradiation-induced defect sink structure was significant, but only for the lowest irradiation temperatures. A detailed discussion of these arguments can be found elsewhere [32].

More recently, Wiedersich $[14,20]$ has examined the possible role of defect clusters in enhancing intercascade recombination, and therefore their role in reducing the number of freely-migrating defects. He argued that computer simulations indicate that only about one-half of the additional decrease arises from intracascade annealing at elevated temperatures, but that the larger observed decrease can be rationalized in terms of uncorrelated defect annihilation at previously produced clusters. As a consequence of the stronger clustering of vacancies than interstitials within cascades, an excess of freely-migrating interstitials arrives at the clusters, which causes vacancy clusters to shrink (even without thermally-activated vacancy emission) and interstitial clusters to grow. Subsequent cascades can also disperse pre-existing clusters. Hence the clusters will have a finite lifetime, and a quasi-steady state will develop during irradiation that includes a distribution of cluster sizes and an excess concentration of freely-migrating interstitials over vacancies. Because of their finite lifetime, no lasting microstructural features such as, for example, radiation-induced precipitation, will persist at any individual cluster site. 
Clearly, the key to understanding the effect of defect clusters on intercascade defect annihilation is knowledge of the cluster lifetimes and their number densities as a function of irradiation conditions, particularly irradiation temperature and primary recoil spectrum. In particular, a model would need to explain the experimental observations of the broad temperature range $\left(400-700^{\circ} \mathrm{C}\right)$ over which the freely-migrating defect concentrations appear to be independent of temperature, as well as the strong functional dependence on recoil energy seen in Fig. 2.

Using the effective medium approach developed by Brailsford and Bullough [33], the sink strengths and lifetimes of the clusters can be calculated from standard rate theory. Details of the formulation, and a discussion of the simplifying assumptions that are required, can be found elsewhere [14j. Essentially, all defects are assumed to be generated at a single cascade size; interstitial loops that grow larger than the cascade diameter become part of the dislocation network; the vacancies in loops that shrink below a minimum size are added to the freely-migrating defect concentration; the sink strengths are calculated for spherical sinks in an effective medium; and thermal evaporation of defects from clusters is neglected. The last assumption implies that the resilts are independent of defect-production rate so long as comparisons are made on the basis of dose rather than time. This is because in the steady-state approximation, where recombination terms cancel, the differential flux of interstitials and vacancies to clusters is proportional to the defect production rate and independent of temperature. The cluster growth (shrinkage) rate is therefore also proportional to the defect production rate, and independent of temperature, as is the dissolution rate by subsequent cascades. Hence the development of the cluster population becomes only a function of the product of displacement-rate and time, i. e., the dose.

The calculated cluster concentrations exhibit maxima at the size input for production. This occurs as a consequence of cascade dissolution. During the time required to shrink a vacancy cluster (or to grow an interstitial cluster), more cf the "older" clusters are lost by cascade dissolution. Hence similar distributions occur for a wide range of assumed production sizes, and the sink strength from clusters is dominated by clusters near their size of formation.

The total calculated sink strength, and the fractions due to the interstitial and vacancy clusters, are shown in Fig. 4 as a function of the assumed cluster size at formation, $\mathbf{n}_{\mathbf{0}}$. At larger sizes, the sink strength depends only weakly on cluster (cascade) size. The sink strength, however, becomes larger for smaller clusters. Note that although the background sink strength was chosen to be very high $\left(\mathrm{p}_{0}=10^{-3}\right.$, which corresponds to a dislocation density of $\left.\sim 10^{12} \mathrm{~cm}^{-2}\right)$ for the calculations, the cluster contribution $\left(>10^{-2}\right)$ overwhelms this background by more than an order of magnitude. The largest contribution comes from the (smaller but more numerous) interstitial clusters; the vacancy clusters contribute about one-third. Doses between 1-5 dpa are required to reach quasisteady state. However, since the sink strength is dominated by the small interstitial and large vacancy clusters, the sink strength approaches its saturation value at substantially smaller doses. The general features of these calculations are relatively robust for variations of the input parameters within physically reasonable ranges [14].

The very large sink strengths indicated in Fig. 4 imply that much of the additional decrease (Figs. 1 and 2) seen in freely-migrating defect production efficiency results 
from intercascade annihilation at vacancy and interstitial loops produced directly in high-energy cascade events. The effects of intracascade recombination and of cluster sinks on the average number of jumps a defect makes between its creation and annihilation are illustrated further in Fig. 5, where this number is plotted (for several cascade sizes) as a function of dose. As shown, isolated defects would make an average of 1000 jumps before annihilation under the assumed matrix sink strength. The developing cluster density reduces the defect lifetime, and therefore the average number of jumps, from the recombination within cascades value, quite rapidly with dose. Only 0.1-0.2 dpa are required to produce an almost order of magnitude reduction in the average number of defect jumps.

\section{CURRENT STATUS AND FUTURE OUTLOOK}

During the past decade, considerable progress has occurred in our fundamental understanding of freely-migrating defect concentrations. Several experiments have provided definitive evidence that the efficiency for producing freely-migrating defects decreases strongly with increasing primary recoil energy [4]. Systematic studies [8-10] have shown that the net decrease is substantially greater than that found for the production of stable defects during irradiation to low doses at cryogenic temperatures.

Because of this strong reduction at high recoil energies, dpa calculations are inadequate for correlating microstructural changes in many irradiation environments. However, the semi-quantitative understanding of freely-migrating defect production that has emerged does provide a physically realistic basis for deriving such correlations. This has been demonstrated most recently by the success of Mansur and Farrell [5] in accounting for the rate of embrittlement observed at the HFIR pressure-vessel wall, and the success of Heinisch [25] in correlating mechanical property changes in neutronirradiated steels, both on the basis of strong primary recoil effects on freely-migrating defect fractions.

Only about $1 \%$ of the number of displaced atoms calculated for energetic recoil atoms actually escape and undergo free migration. Currently two explanations have been proposed for this small fraction relative to the $-25 \%$ found for defect survival at low temperatures. Certainly part of the additional losis is due to thermally-activated defect motion, and therefore increased intracascade defect annihilation ard clustering, at elevated temperatures. However cascade remnants, as briefly reviewed above, may provide highly effective sinks for intercascade defect annihilation. The relative contributions from each mechanism are currently uncertain.

It appears that more vacancies than interstitials cluster within energetically dense displacement cascades. In this case, the fraction of freely-migrating interstitial atoms will exceed that of vacancies. The term "radiation-enhanced" diffusion implies that the consequences of diffusion remain the same during irradiation as during thermal annealing, where vacancies typically constitute the only significant mobile species. Another common assumption made in using rate theory is that the individual contribution from vacancy and interstitial defects to diffusion is identical. Interstitials, with for example their tendency to interact more strongly with solute elements, violate this criterion. Clearly many of these long-held concepts need to be reconsidered in light of the mounting evidence that $F_{i}$ is not equal to $F_{v}$. Of course at sufficiently high tempera- 
tures, where none of the cascade clusters are thermally stable, these probabilities must become equal.

The most important remaining question, at least for correlations in technologically important radiation environments, is the absolute concentration of freely-migrating defects at recoil energies ( $210 \mathrm{keV}$ ) typical of fission and fusion reactors. The error bars on the existing determinations of freely-migrating defect fractions are large. For example, the absolute values obtained by the Berlin group from fits to their data fall in the range from 0.003 to .06 , an uncertainty of more than an order of magnitude. A similar degree of uncertainty, at least at the higher recoil energies, can be seen in the relative efficiencies determined at Argonne. The reason for the large uncertainty at present is readily identified. Because the escaping fractions are so small, it is difficult to measure them accurately. Therefore, more quantitative studies are necessary, particularly in neutron environments with their characteristically high, and often narrower, primary recoil spectrum.

Also highly desirable would be a deconvolution of the freely-migrating defect fractions, which are currently expressed in terms of the median recoil energy, into a direct function of the primary knock-on energy. This would permit a more accurate identification of when subcascade formation becomes a significant factor in the defect production process, and serve as a more direct input for modeling calculations. Deconvolutions of low-temperature defect survival measurements have been performed, although large uncertainties can arise $[7,18]$. It should therefore be recognized that much less quantitative information is available on freely-migrating defect production. Thus the successful completion of this task awaits more extensive experimental results. Neutron irradiation studies, again because of their often characteristically narrower range of recoil energies, would be particularly useful in this effort.

\section{REFERENCES}

1) Simons, R. L.: J. Nucl. Mats. 1986, 141-143, 665.

2) Proceedings of the Workshop "Effects of Recoil Energy Spectrum and Nuclear Transmutations on the Evolution of Microstructure", Eds. Green, W. V., Victoria, M., Leffers, T., and Singh, B. N.: Radiation Effects and Defects in Solids, 1990, 113.

3) Proceedings of the International Workshop "Radiation Damage Correlation for Fusion Conditions", Eds. Singh, B. N., Horsewell, A., Victoria, M., and Green, W. V.: J. Nucl. Mats. 1990, 174(2\&3).

4) Rehn, L. E. and Okamoto, P. R.: Mater. Sci. Forum, 1987, 15-18, 985.

5) Mansur, L. K. and Farrell, K.: J. Nucl. Mater., 1990, 170, 236.

6) Averback, R. S., Benedek, R. and Merkle, K. L.: Phys. Rev., 1978, B18, 4156.

7) Kinney, J. H., Guinan, M. W. and Munir, Z. A.: J. Nucl. Mater., 1984, 122/123, 1028.

8) Rehn, L. E., Okamoto, P. K. and Averback, R. S.: Phys. Rev., 1984, B30, 3073.

9) Hashimoto, T., Rehn, L. E. and Okamuto, P. R.: Phys. Rev., 1988, B38, 12865.

10) Erck, R. A. and Rehn, L. E.: J. Nucl. Mater., 1989, 168, 208.

11) Summary of the Workshop "Radiation Effects on Materials in High-Radiation Environments", Eds. Weber, W. J., Mansur, L. K., Clinard, Jr., F. W., and Parkin, D. M.: J. Nucl. Mater., 1991, in press. 
12) Nanstod, R. K., Farrell, K., Broski, D. N. and Corwin, W. R.: J. Nucl. Mater., $1988,158,1$.

13) Naundorf, V. and Wollenberger, H.: in Ref. 3, p. 141.

14) Wiedersich, H.: this conference.

15) Goland, A.: J. Nucl. Mater., 1979, 85/86, 453.

16) Averback, R. S. and Seidman, D. N.: Mater. Sci. Forum, 1987, 15-18, 963.

17) Markle, K. L.: Phys. Status Solidi, 1966, 18, 73.

18) Jung, P., Nielsen, B. R., Anderson, H. H., Bak, J. F., Knudsen, H., Coltman, Jr., R. R., Klabunde, C. E., Williams, J. M., Guinan, M. W. and Violet, C. E.: Effects of Radiation on Materials, Brager, H. R. and Perrin, J. S., eds. (American Society for Testing and Materials, Philadelphia, PA, 1982) 963.

19) Wiedersich, $\mathrm{H}$ : Physics of Radiation Effects in Crystals, Johnson, R. H. and Orlov, A.N., eds. (Elsevier Science Publishers, London, 1986) 225.

20) Wiedersich, H: in Ref. 1, p. 97.

21) Wollenberger, H: Nucl. Instr. and Meth., 1990, B48, 493.

22) Müller, A., Naundorf, V. and Niacht, M.-P.: J. Appl. Phys., 1988, 64, 3445.

23) Müller, A., Naundorf, V. and Macht, M.-P.: J. Nucl. Mater., 1988, 155-157, 1128.

24) Murphy, S. M. and Periss, J. M.: J. Nucl. Mater., 1990, 171, 360.

25) Heinisch, H. L.: J. Nucl. Mater., 1991, 178, 19.

26) Kinchin, G. H. and Pease, R. S.: Rep. Prog. Phys., 1955, 18, 1.

27) Norgett, M. J., Robinson, M. T. and Torrens, I. M.: Nucl. Engrg. Des., 1974, 33, 50.

28) Beeler, Jr., J. R., Beeler, M. F. and Parks, C. V.: Proceedings of the Conference on Radiation Effects and Tritium Technology for Fusion Reactors, Gatlinburg. Tennessee. 1975, Watson, J. S. and Wiffen, F. W., eds. (ERDA, Oak Ridge, Tenn., 1976) p. 358.

29) Robinson, M. T. and Torrens, I. M.: Phys. Rev. B, 1974, 9, 50 n8.

30) Guinan, M. W. and Kinney, J. H.: J. Nucl. Mater., 1981, 103,:104, 1319.

31) Diaz de la Rubia, T., Averback, R. S., Benedek, R. and Robertson, I. M.: Rad. Eff. Def. Sol., 1990, 113, 39.

32) Rehn, L. E.: in Ref. 2, p. 144.

33) Brailsford, A. D. and Bullough, R.: Phil. Trans. Royal Soc. London, 1981, 302, 87. 


\section{FIGURE CAPTIONS}

Fig. 1. Measured efficiencies for producing Frenkel pairs at $4 \mathrm{~K}$ in $\mathrm{Cu}$ for electrons (circles), ions (triangles), and neutrons (squares), plotted as a function of the weighted-average recoil energy.

Fig. 2. Measured relative efficiencies for producing freely-migrating defects in Ni(Si)solid squares, $\mathrm{Cu}(\mathrm{Au})$-open triangles, and $\mathrm{Mo}(\mathrm{Re})$-open squares alloys during ion irradiation plotted as a function of the weighted-average recoil energy.

Fig. 3. Log-log plot of absolute efficiencies of various ions for producing freelymigrating defects in $\mathrm{Ni}$ as a function of the weighted-average recoil energy (top); as a function of the nuclear stopping power (bottom). From Ref. 13.

Fig. 4. Results of model calculations for total sink strength as a function of cascade size (squares), as well as for interstitial clusters (triangles) and vacancy clusters (circles) at a dose of $1 \mathrm{dpa}$.

Fig. 5. Total number of jumps per defect calculated (see text) with and without cluster sinks for several cascade sizes between $\mathbf{4 0}$ and $\mathbf{4 0 0}$. 


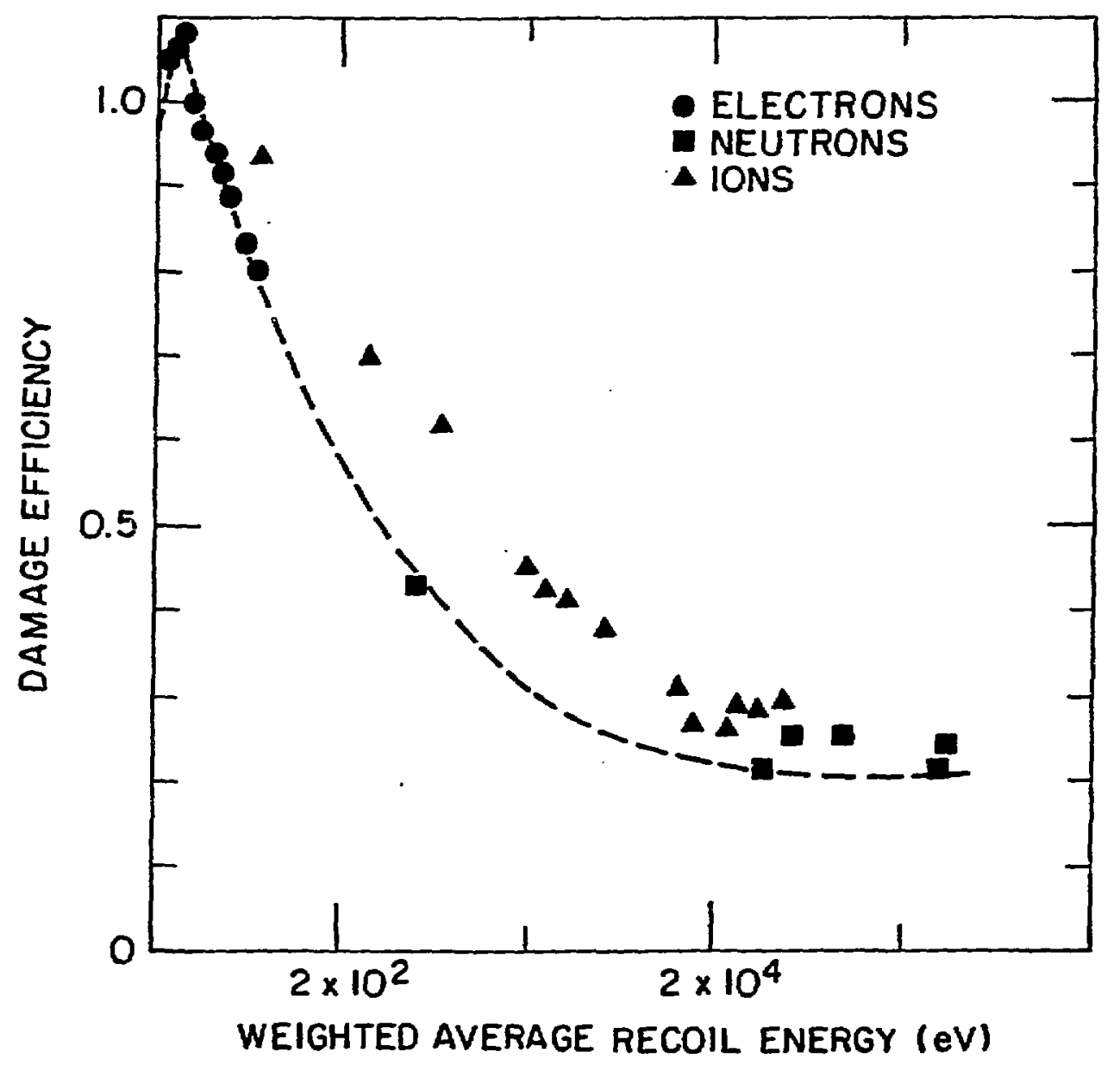

Fig. I 
$\tau \cdot]$

RELATIVE EFFICIENCY

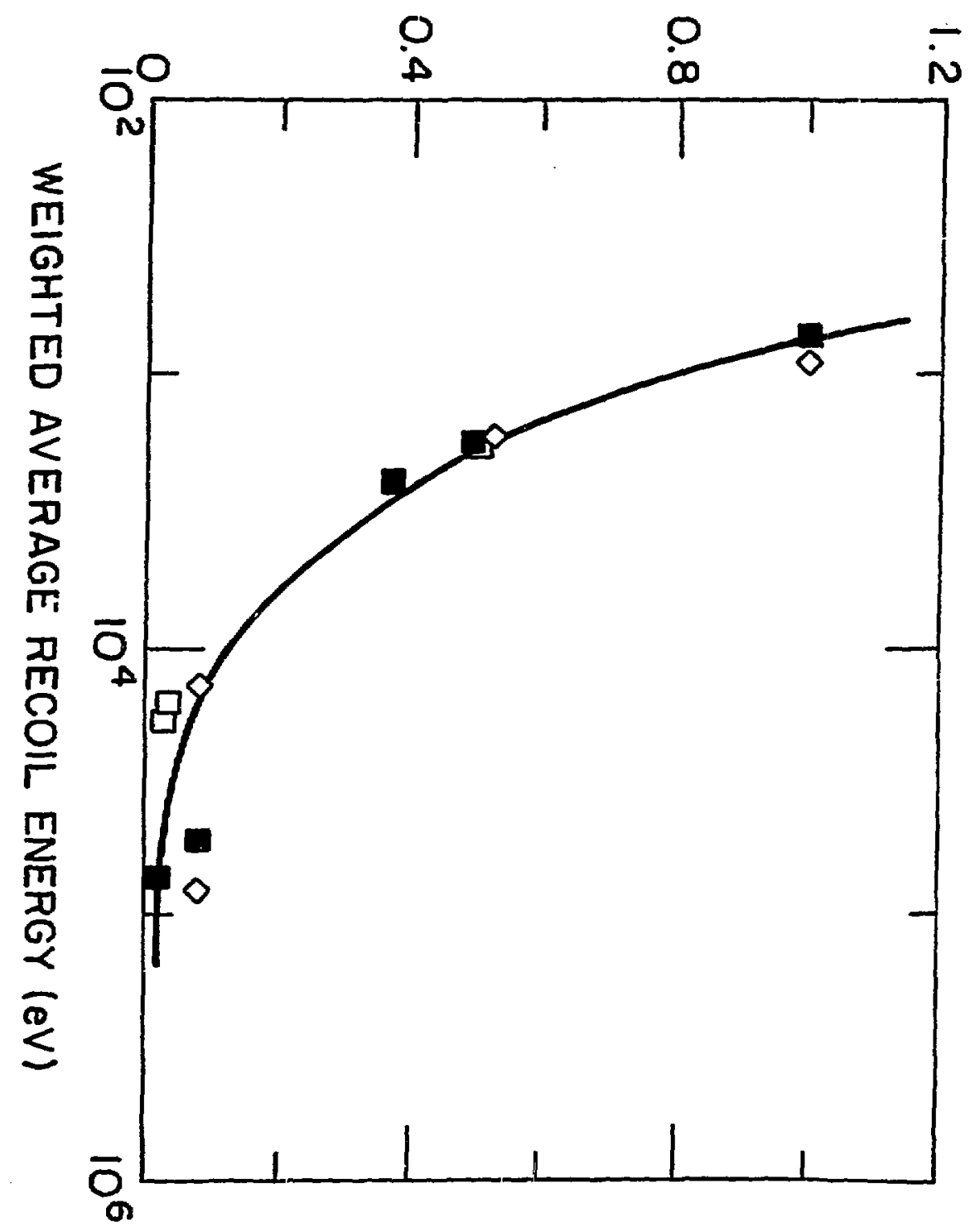




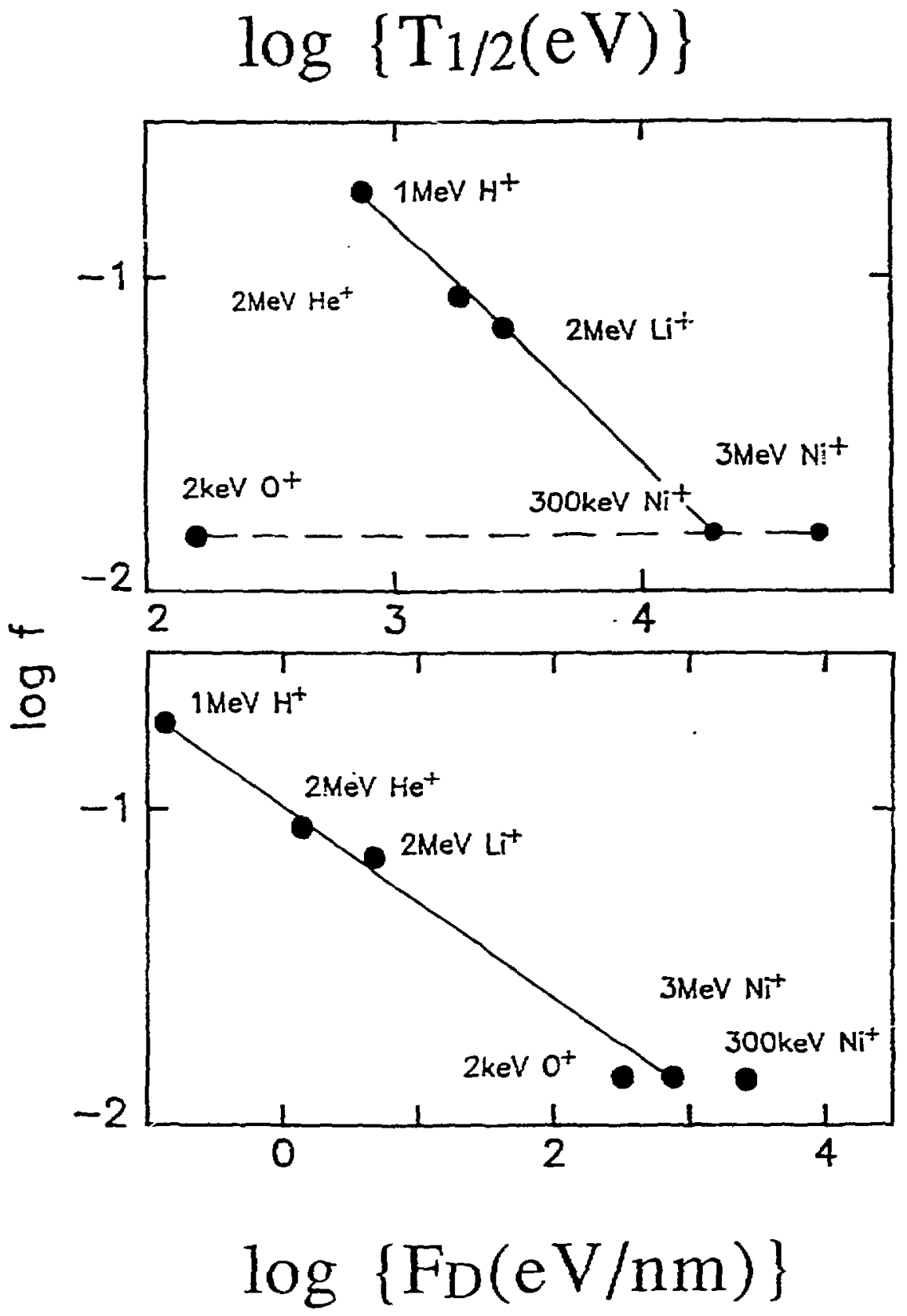


Sink strength, annihilations/defect jump

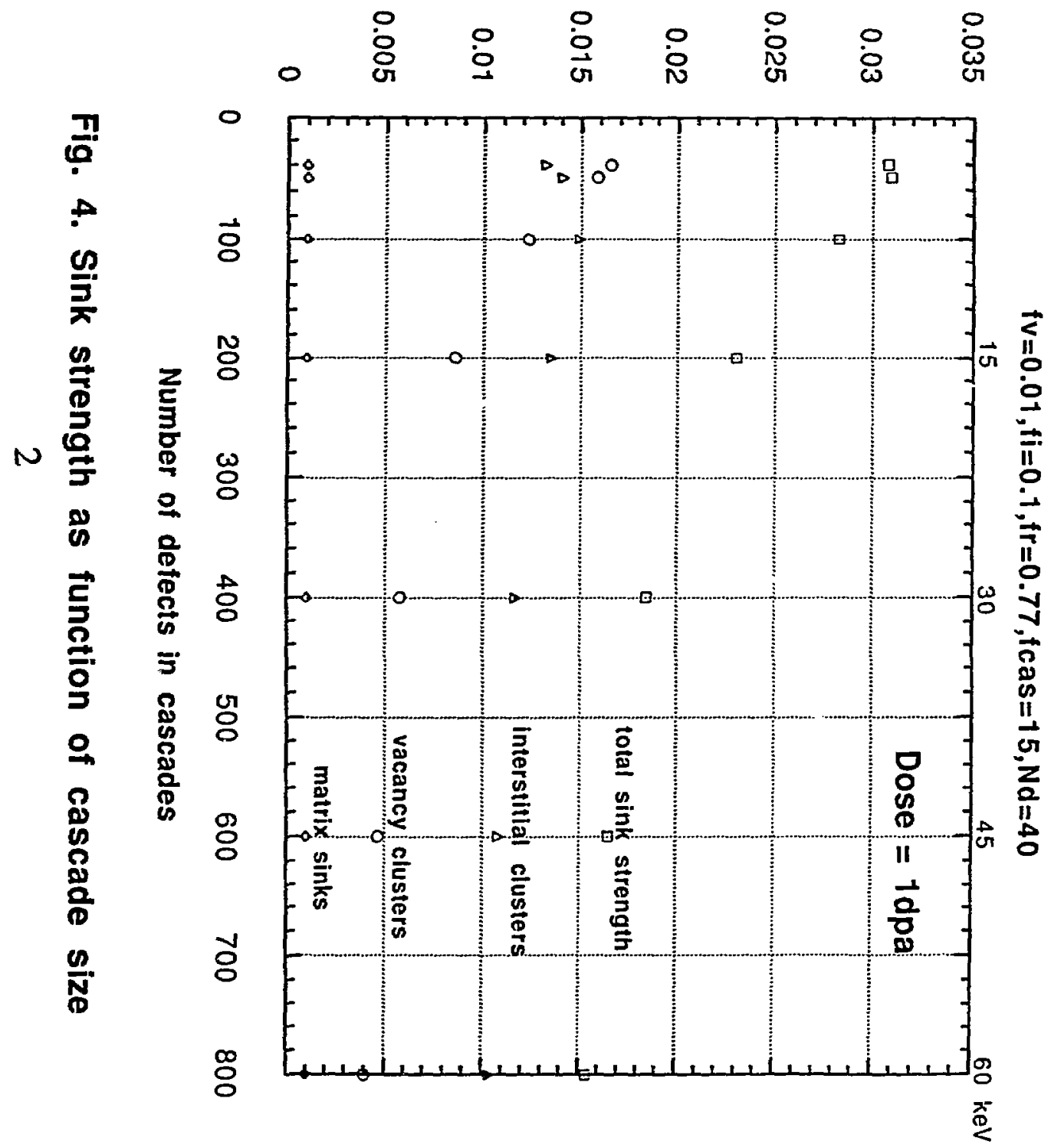




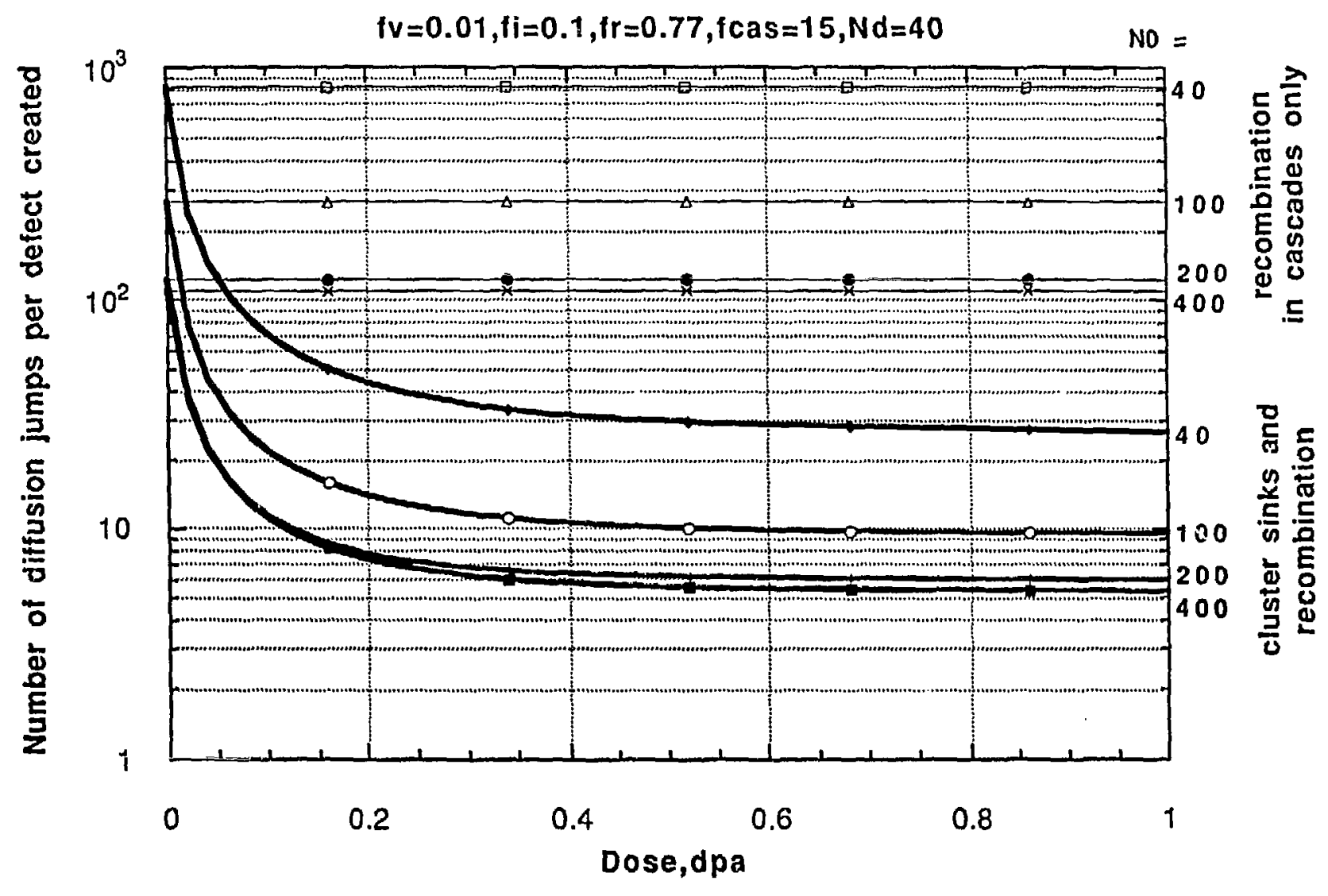

Fig. 5. Diffusion with and without cluster sinks for several cascade sizes, NO 